\title{
Effect of the Dog positioning on X-Ray Table on Hip Dysplasia Parameter Evaluation
}

\author{
João Martins ${ }^{1,2}$ Bruno Colaço ${ }^{2,3}$ Sofia Alves-Pimenta ${ }^{2,30}$ \\ António J. Ferreira ${ }^{5}$ Mário M. Ginja2,6 \\ ${ }^{1}$ Faculty of Veterinary Medicine, Lusófona University, Lisboa, Portugal \\ ${ }^{2}$ CITAB - Centre for the Research and Technology of Agro- \\ Environmental and Biological Sciences, Vila Real, Portugal \\ ${ }^{3}$ Department of Animal Science, University of Trás-os-Montes and \\ Alto Douro, Vila Real, Portugal \\ ${ }^{4}$ Department of Veterinary Medicine, Surgery and Anatomy, Faculty \\ of Veterinary Medicine, University of León, Spain \\ ${ }^{5}$ CIISA - Department of Clinics, University of Lisbon, Lisbon, Portugal \\ ${ }^{6}$ Department of Veterinary Science, University of Trás-os-Montes and \\ Alto Douro, Vila Real, Portugal
}

Address for correspondence Mário M. Ginja, DVM, PhD, Department

Vet Comp Orthop Traumatol 2019;32:376-382.

of Veterinary Science, Trás-os-Montes and Alto Douro University, 5001-801 Vila Real, Portugal (e-mail: mginja@utad.pt).

\begin{abstract}
\section{Keywords}

- Norberg angle

- subluxation index

- subluxation category

- pelvic tilting

- femoral rotation

Objective The aim of this study was to evaluate the effect of pelvic tilting along the long axis and femoral rotation on Norberg angle (NA), subluxation index (SI) and subluxation category (SC) in the standard ventrodorsal hip extended (VDHE) radiographical view on live animals.

Study Type This was a retrospective clinical study.

Materials and Methods Pairs of VDHE views, one adequately positioned and the other with pelvic tilting or femoral internal or external rotation, were compared for the NA, SI and SC.

Results On the malpositioned pelvis set, on the underside the mean \pm SD NA was $98.7 \pm 6.1^{\circ}$, the SI was $0.27 \pm 0.12$ and the $S C$ was $2.8 \pm 0.8$ versus, on the acceptable set, the NA was $99.2 \pm 6.4^{\circ}(p>0.05)$, the SI was $0.25 \pm 0.12(p<0.05)$ and the SC was $2.3 \pm 0.9(p<0.05)$; on the malpositioned upperside, the NA was $102.1 \pm 6.4^{\circ}$, the $\mathrm{SI}$ was $0.21 \pm 0.14$ and the SC was $1.7 \pm 1.1$ versus, on the acceptable positioned set, the NA was $100.8 \pm 6.7^{\circ}(p<0.05)$, the SI was $0.24 \pm 0.15(p<0.05)$ and the SC was $2.3 \pm 1.2(p<0.05)$. Femoral internal or external rotation sets did not show significant differences between malpositioned and acceptable positioned sets $(p>0.05)$.

Conclusions In clinical practice, pelvic tilting along the long axis in VDHE view results in non-favourable hip changes in the NA, SI and SC on the underside and favourable on the upperside, and the internal or external femoral rotation did not affect these variables.
\end{abstract}

(1) Sofia Alves-Pimenta's ORCID is https://orcid.org/0000-0001-

9842-1759.

received

October 24, 2018

accepted after revision

April 2, 2019 (c) 2019 Georg Thieme Verlag KG Stuttgart · New York
DOI https://doi.org/

10.1055/s-0039-1688991.

ISSN 0932-0814. 


\section{Introduction}

Canine hip dysplasia is the most prevalent developmental orthopaedic disease affecting dogs worldwide. ${ }^{1}$ Canine hip dysplasia has a determinant hereditary component; however, a marker-assisted accurate diagnostic test is still lacking, due to its complex polygenic nature. ${ }^{2}$ Since 1960 the standard ventrodorsal hip extended (VDHE) radiographic view remains the recommended worldwide technique for hip dysplasia screening. ${ }^{3,4}$ Canine hip dysplasia diagnosis is essential for the selection of breeding stock to reduce genetic transmission to offspring. $2,5,6$

The Norberg angle (NA), hip subluxation and/or congruity are parameters used worldwide to score hip dysplasia grades: the Fédération Cynologique Internationale (FCI), the Orthopedic Foundation for Animals and the British Veterinary Association/ Kennel Club (BVA/KC) ${ }^{7-10}$ These hip evaluation parameters are especially important to score normal joints or in cases that do not yet show osteoarthritic changes, such as deformation of the neck and head of the femur or acetabulum. ${ }^{1,9}$ Dog breeders use hip dysplasia scoring to select animals with better hips for breeding. ${ }^{1}$ Inappropriate positioning on the X-ray table is associated with a poor technical quality of the VDHE view and altered projected relationship between the acetabulum and femoral head. ${ }^{11}$ Pelvic malpositioning can be a result of tilting along its long or short axis. ${ }^{11-13}$ Previous studies on cadavers have shown that longitudinal pelvic and femoral internal or external rotation are directly associated with inadequate NA, femoral head subluxation index (SI) and femoral head subluxation category (SC) measurements. ${ }^{12,14}$ Other studies, performed using a bone model ${ }^{13}$ and computed tomography simulation, ${ }^{15}$ obtained contradictory results in the association of pelvic tilting along the short axis with NA measurements.

Although some reports on cadavers investigated the effects of dog malposition in canine hip dysplasia scoring, ${ }^{12,14}$ no studies that quantify the effect of pelvic tilting and femoral rotation on VDHE radiographic views in live animals are available. The main purpose of this study was to evaluate the relationship between pelvic tilting along the long axis or femoral rotation and the measurements of the $\mathrm{NA}$, the SI and the SC on VDHE view.

\section{Materials and Methods}

\section{Animals}

This was a retrospective multi-centre study based on the analyses of 248 radiographs of dogs that underwent screening for canine hip dysplasia using VDHE view between 2003 and 2018. Radiographs were performed at the Veterinary Teaching Hospitals of University of Trás-os-Montes and Alto Douro and University of Lisbon, and Veterinary Hospital of Montenegro (Portugal). Recorded data included breed, age at time of the radiography, sex, bodyweight and place of evaluation. The inclusion criteria were pairs of VDHE views, one with acceptable positioning and other with malpositioning in terms of pelvic tilting along the long axis or femoral internal or external rotation, at concurrent time points, of dogs older than 12 months of age. All views in the acceptable set had adequate technical quality for canine hip dysplasia scoring, with maximum pelvic tilting ${ }^{16}$ of 3 degrees and patellar medial or lateral displacement indices ${ }^{14} \geq 0.40$, decided by mutual agreement between MMG and AJF, radiologists with large experience. Due to the observational nature of the study, the ethical committee approval and the owner consent were waived.

Radiographs were obtained with deep sedation using medetomidine (Domitor: Orion Corporation, Espoo, Finland) and butorphanol (Torbugesic Injectable: Fort Dodge Veterinaria, Girona, Spain) intravenously and reversed with atipamezole hydrochloride (Antisedan: Orion Corporation, Espoo, Finland) intramuscularly. The VDHE views were obtained with dogs placed in dorsal recumbency on the X-ray table, with the hindlimbs extended parallel to each other and to the table top as much as possible, and the stifles internally rotated. ${ }^{1,7}$

\section{Radiographical Examination}

First, the pelvic rotation was evaluated measuring in $\mathrm{mm}$ the right and left iliac horizontal diameter (IHD) as described previously: a straight line was drawn on each side between the dorsal and ventral iliac cortex at the level of the cranial aspect of sacroiliac joint. ${ }^{16}$ The regression equation $\mathrm{y}$ $=0.997 \mathrm{x}+0.06$ was used to estimate the degrees of pelvic rotation ( $\mathrm{y}$ ) based on IHD asymmetry in $\mathrm{mm}(\mathrm{x}) .^{16}$ The underside and upperside were defined for each animal based on malpositioned views to estimate the improvement of rotation in degrees of acceptable positioned views (-Fig. 1).

The patellar displacement over the femoral diaphysis in the acceptable positioned and malpositioned femoral rotation views was used to evaluate femoral internal and external rotation as described previously. ${ }^{14}$ For this purpose, a line was drawn from the base to the apex of the patella, then the horizontal distance between distal femoral metaphysis cortex (metaphysis thickness) and between the patellar line to the lateral (external femoral rotation set) and medial femoral cortex (internal femoral rotation set) was measured in millimetres. Then the patellar medial or lateral displacement indices were calculated. The difference between normal positioned patellar index and malpositioned patellar index $(\mathrm{x})$, and the regression equations $\mathrm{y}=55.7 \mathrm{x}+3.2$ or $\mathrm{y}=61.6$ $\mathrm{x}+2.6$ were used to estimate femoral internal or external rotation impairment (y) in degrees respectively (-Fig. 2). ${ }^{14}$

Canine hip dysplasia parameters were evaluated measuring the NA, the SI and the SC. ${ }^{12,14}$ The NA was measured in degrees between a line joining the centres of the femoral heads and the line joining the centre of the femoral head and the craniolateral aspect of the acetabular rim. ${ }^{17,18}$ The SI was measured dividing the distance between the centre of the femoral head and the acetabular centre by the radius of the femoral head. ${ }^{19}$ The SC was assessed in one of seven categories, from 0 to femoral head centred in acetabulum to 6 -femoral head centre completely dislocated from acetabulum. ${ }^{10,12}$

Until 2010 the images were obtained on hard copy radiographs and later by computed digital radiography. Hard copy radiographs were digitalized at a resolution of 200 dpi using a computer scanner (ScanMaker 9800XL; Microtek, Carson, California, United States) provided with a transparency adapter. Digital images were grouped on eight sets to be analysed, 

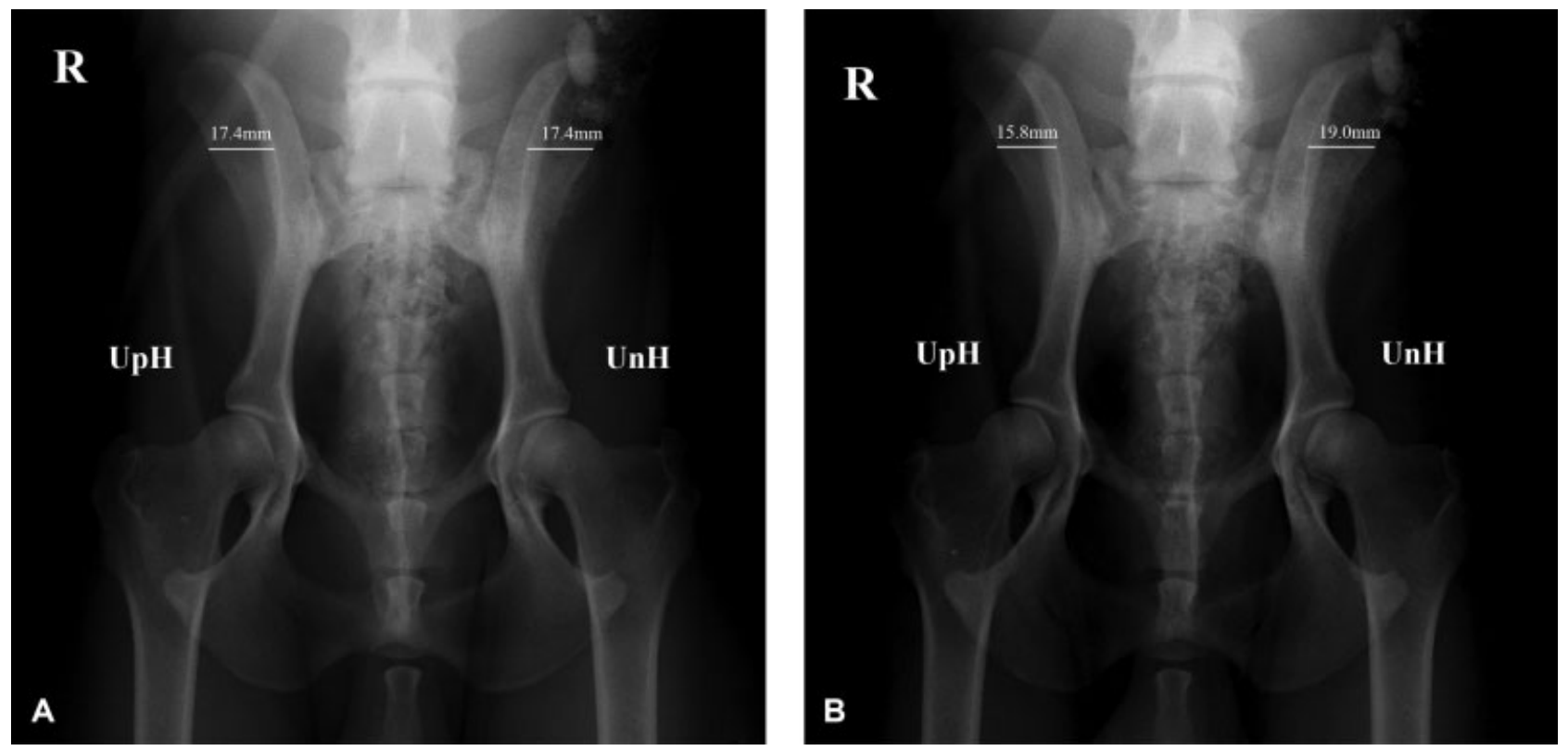

Fig. 1 (A) Ventrodorsal pelvis acceptable positioned view without pelvic tilting. (B) Ventrodorsal pelvis malpositioned view (same animal of A) with pelvic tilting to the left side, asymmetry of $3.2 \mathrm{~mm}$ in iliac horizontal diameter corresponding to a pelvic tilting of $3.3^{\circ}$ to the left side. R, right side; UnH, underside hip; UpH, upperside hip.
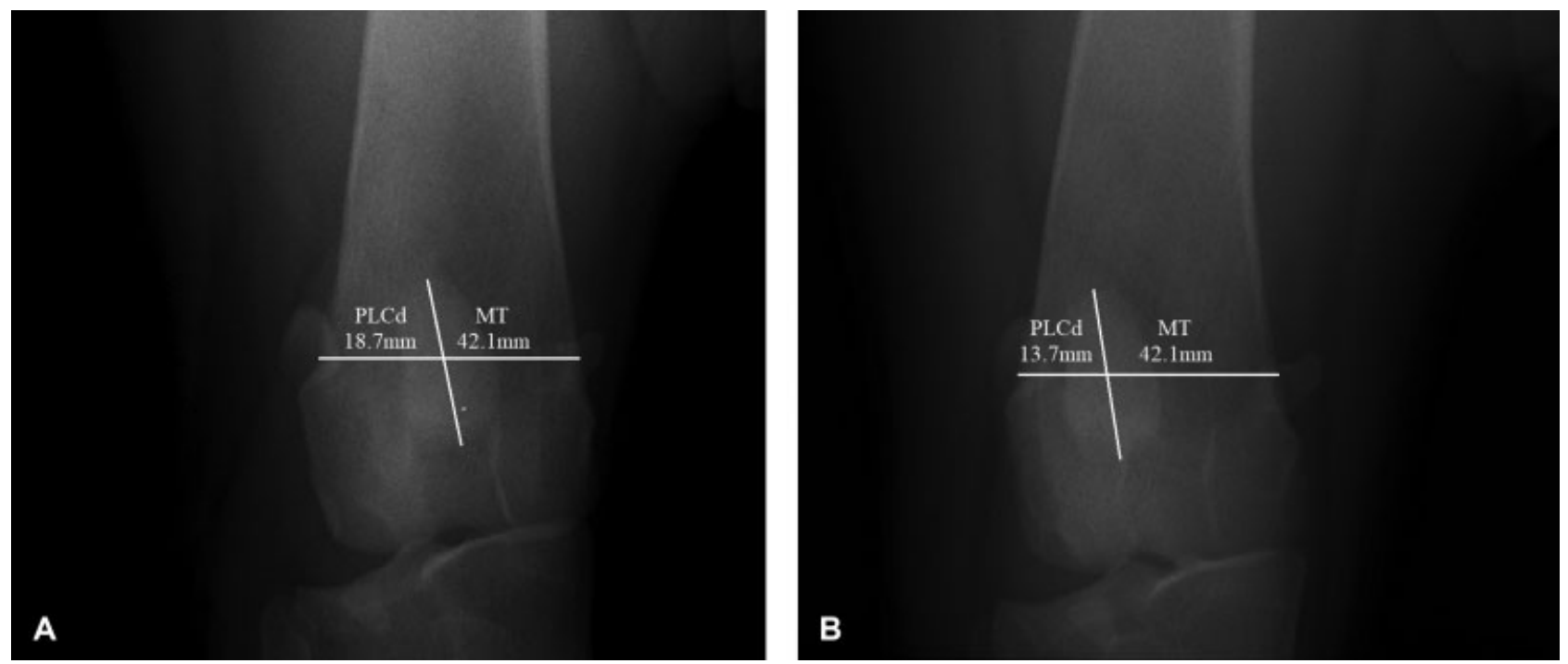

Fig. 2 (A) Craniocaudal acceptable right femoral view. (B) Craniocaudal malpositioned right femoral view (same animal of A) with lateral rotation. Patellar lateral displacement index of 0.44 in $A$ and 0.33 in B, corresponding to a difference of 0.11 in patellar lateral displacement femoral index and an improvement in lateral patellar positioning in A of $9.4^{\circ}$. MT, metaphysis thickness; PLCd, patellar lateral cortex distance.

acceptable positioned and malpositioned: pelvis underside and upperside, femoral external and internal rotation. The positioning analyses measurements were performed by JM and the hip dysplasia parameters NA, the SI and the SC by MMG, on randomly chosen digital images of each set using the software OSIRIS (OSIRIS Imaging Software Version 3.1: University Hospital of Geneva, Geneva, Switzerland).

\section{Statistical Analysis}

Statistical analysis was performed using the computer software SPSS (SPSS Statistics for Windows Version 23.0; IBM Corp., Armonk, New York, United States). Descriptive statis- tics were computed for all variables. The data analysis was performed on joints individually, by grouping data eight sets of four pairs, the acceptable positioned VDHE views versus the respective malpositioned pair: underside and upperside (pelvic tilting); external femoral rotation; internal femoral rotation. The paired $t$-test was used to evaluate if the NA, the SI and the SC were similar. The SC variable was directly converted into a numeric scale and evaluated as continuous. ${ }^{9,12}$ The $p<0.05$ was considered to be significant. In significant differences, the mean standard deviation of Cohen ( $d$ Cohen) was used to measure the effect size: small $\geq 0.20$, medium $\geq 0.50$ and large $\geq 0.80 .^{20}$ 
Table 1 Norberg angle, subluxation index and subluxation category in malpositioned and acceptable positioned pelvic sets

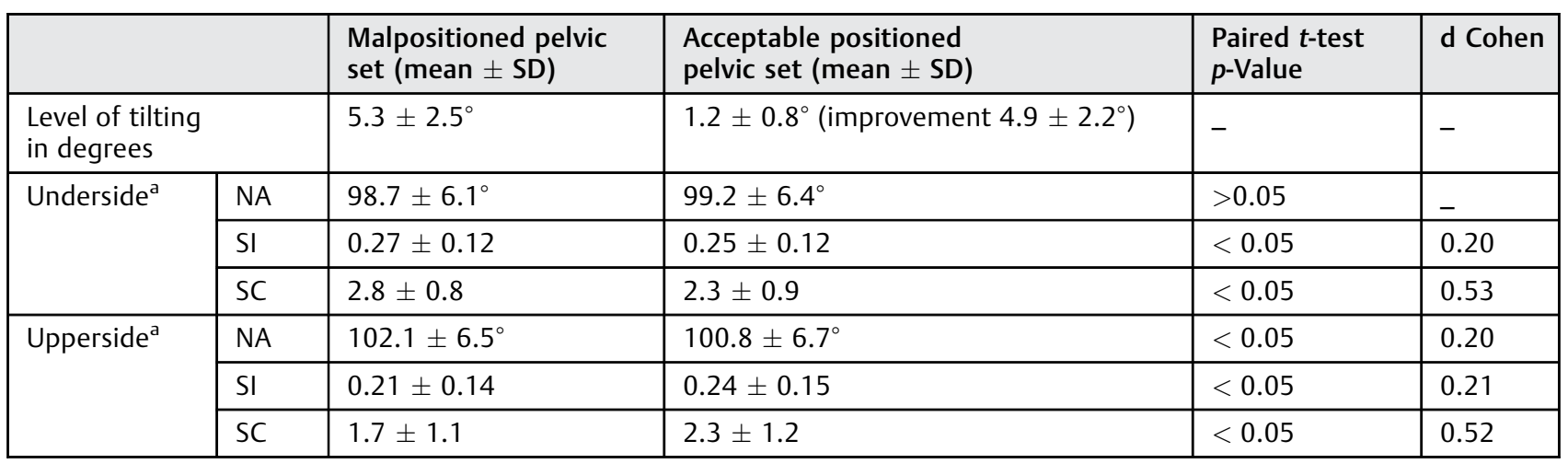

Abbreviations: d Cohen, mean standard deviation of Cohen; NA, Norberg angle; SC, subluxation category; SD, standard deviation; SI, subluxation index. ${ }^{a}$ The side of the joint in the malpositioned view was used as reference to define in acceptable positioned view pair, the right or left side as underside or upperside.

\section{Results}

Two-hundred and forty-eight VDHE views were available providing 124 pairs of acceptable positioned versus malpositioned radiographs from 124 dogs. One hundred and two radiographs from 51 dogs were compared for malpositioned underside hips versus the acceptable position and 51 malpositioned upperside hips versus the acceptable position. Seventyeight views from 39 dogs were compared for 58 internal femoral rotation hips versus acceptable femoral position. Sixty-eight views from 34 dogs compared for 48 external femoral rotation hips versus acceptable femoral position.

The most prevalent breeds were Estrela Mountain dog (62.9\%, 78/124), and Portuguese Pointing dogs (16.1\%, 20/ $124)$. Females $(70.2 \%, 87 / 124)$ were in greater number than males $(29.8 \%, 37 / 124)$. The mean bodyweight was $37.2 \pm 9.4 \mathrm{~kg}$ and the mean age $25.8 \pm 16.5$ months.

\section{Pelvic Tilting Sets}

On the acceptable positioned pelvis set, the mean \pm standard deviation (SD) of pelvic tilting was $1.2 \pm 0.8^{\circ}$; in $2 / 51$ of cases there was no rotation, 30/51 kept the rotation side and $19 / 51$ of cases changed the rotation side of malpositioned view. Despite this asymmetry in the acceptable positioned pelvis set, there were no significant differences between underside and upperside (using as reference the malposition side), in any of the variables NA, SI or SC $(p>0.05)$. The improvement in pelvic rotation on acceptable set ranged from 1.4 to $11.0^{\circ}$, mean \pm SD $4.9 \pm 2.2^{\circ}$.
On underside of malpositioned pelvis set, the mean \pm SD $\mathrm{NA}$ was $98.7 \pm 6.1^{\circ}$, the SI was $0.27 \pm 0.12$ and the SC was $2.8 \pm 0.8$ versus, on same joints in acceptable positioned set, the NA was $99.2 \pm 6.4^{\circ}(p>0.05)$, the SI was $0.25 \pm 0.12$ $(p<0.05)$ and the SC was $2.3 \pm 0.9(p<0.05)$. The parameter that showed a bigger size effect was the SC ( - Table 1 ).

On upperside malpositioned pelvis set, the mean \pm SD NA was $102.1 \pm 6.5^{\circ}$, the SI $0.21 \pm 0.14$ and the SC $1.7 \pm 1.1$ versus, on the same joints in the acceptable positioned set, the NA was $100.8 \pm 6.7^{\circ}(p<0.05)$, the SI was $0.24 \pm 0.15$ $(p<0.05)$ and the SC was $2.3 \pm 1.2(p<0.05)$ The parameter that showed a bigger size effect was the SC ( - Table 1 ).

\section{Internal Femoral Rotation Set}

In the malpositioned internal femoral rotation set, the medial patellar displacement index ranged from 0.14 to $0.47^{\circ}$, mean $\pm S D 0.36 \pm 0.07$, the NA was $103.0 \pm 5.1^{\circ}$, the SI was $0.19 \pm 0.12$ and the SC was $2.1 \pm 0.9$. In the acceptable positioned internal femoral set, the internal femoral displacement index ranged from 0.40 to 0.59 , mean \pm SD $0.49 \pm 0.04$, resulting in an improvement in internal femoral rotation of $10.3 \pm 3.8^{\circ}$. In this set, the NA was $103.7 \pm 4.7^{\circ}(p>0.05)$, the SI was $0.18 \pm 0.11(p>0.05)$ and the SC was $2.1 \pm 0.9(p>0.05)$ (-Table 2$)$.

\section{External Femoral Rotation Set}

In the malpositioned external femoral rotation set, the index of lateral patellar displacement ranged from 0.18 to 0.47 , mean \pm SD $0.32 \pm 0.07$ and the NA was $101.6 \pm 7.2^{\circ}$, the SI

Table 2 Norberg angle, subluxation index and subluxation categories in internal femoral rotation and acceptable positioned sets

\begin{tabular}{|l|l|l|l|}
\hline & $\begin{array}{l}\text { Internal femoral rotation } \\
\text { set (mean } \pm \text { SD) }\end{array}$ & $\begin{array}{l}\text { Acceptable femoral } \\
\text { set (mean } \pm \text { SD) }\end{array}$ & $\begin{array}{l}\text { Paired } t \text {-test } \\
p \text {-Value }\end{array}$ \\
\hline Level of rotation & MPDi $0.36 \pm 0.07$ & $\begin{array}{l}\text { MPDi 0.49 } \pm 0.04 \\
\left(\text { improvement } 10.3 \pm 3.8^{\circ}\right)\end{array}$ & - \\
\hline NA & $103.0 \pm 5.1^{\circ}$ & $103.7 \pm 4.7^{\circ}$ & $>0.05$ \\
\hline SI & $0.19 \pm 0.12$ & $0.18 \pm 0.11$ & $>0.05$ \\
\hline SC & $2.1 \pm 0.9$ & $2.1 \pm 0.9$ & $>0.05$ \\
\hline
\end{tabular}

Abbreviations: MPDi, medial patellar displacement index; NA, Norberg angle; SC, subluxation category; SD, standard deviation; SI, subluxation index. 
Table 3 Norberg angle, subluxation index and subluxation categories in external femoral rotation and normal positioned sets

\begin{tabular}{|l|l|l|l|}
\hline & $\begin{array}{l}\text { External femoral rotation } \\
\text { set (mean } \pm \text { SD) }\end{array}$ & $\begin{array}{l}\text { Acceptable femoral } \\
\text { set (mean } \pm \text { SD) }\end{array}$ & $\begin{array}{l}\text { Paired } t \text {-test } \\
p \text {-Value }\end{array}$ \\
\hline Level of rotation & LPDi $0.32 \pm 0.07$ & $\begin{array}{l}\text { LPDi } 0.47 \pm 0.04 \\
\left.\text { (improvement } 11.7 \pm 4.0^{\circ}\right)\end{array}$ & - \\
\hline NA & $101.6 \pm 7.2^{\circ}$ & $101.7 \pm 7.1^{\circ}$ & $>0.05$ \\
\hline SI & $0.23 \pm 0.11$ & $0.23 \pm 0.11$ & $>0.05$ \\
\hline SC & $2.3 \pm 1.0$ & $2.3 \pm 1.1$ & $>0.05$ \\
\hline
\end{tabular}

Abbreviations: LPDi, lateral patellar displacement index; NA, Norberg angle; SC, subluxation category; SD, standard deviation; SI, subluxation index.

was $0.23 \pm 0.11$ and the SC was $2.3 \pm 1.0$. In the acceptable positioned external femoral set, the external femoral displacement index ranged from 0.4 to 0.55 , mean \pm SD $0.47 \pm 0.04$, resulting in an improvement in external femoral rotation of $11.7 \pm 4.0^{\circ}$. In this set, the NA was $101.7 \pm 7.1^{\circ}$ $(p>0.05)$, the SI was $0.23 \pm 0.11(p>0.05)$ and the SC was $2.3 \pm 1.1(p>0.05)$ (-Table 3$)$.

\section{Discussion}

Despite all the recent research done in molecular diagnosis of canine hip dysplasia, radiographic screening of dog populations for breeding purposes remains the reference method. ${ }^{2}$ However, radiographic scoring is somewhat subjective and affected by inadequate positioning on X-ray table. ${ }^{13}$ The NA, SI and SC are quantifiable and objective parameters used worldwide by the main international canine hip dysplasia scoring systems, including the FCI and BVA/KC. ${ }^{1,10}$ Normal hips should have good femoral head and acetabular congruence resulting in NA $\geq 105^{\circ}$ and very low SI and SC, the ideal being the 0 value. ${ }^{1,10,19}$ Dysplastic hips show additional changes as osteoarthritic signs and deformation of femoral head and acetabulum. ${ }^{9,10}$ Previous studies in cadavers ${ }^{12,14}$ and with simulation of a computer program ${ }^{15}$ have linked pelvic tilting along its long or short axis, and femoral malpositioning, with changes in the relationship between femoral head and acetabulum and consequently in the technical evaluation performed. In this work, only pelvic and femoral tilting along the long body-axis was evaluated because the lack of radiographic superimposition and absence of symmetry of pelvic and femoral bone structures along the long body dorsal plane make it difficult to measure the grade of pelvic tilting along the short axis. ${ }^{13}$

Few VDHE views are perfect with neither pelvic nor femoral rotation. ${ }^{11,16}$ The estimation of the pelvic tilting and femoral level of internal or external rotation based on the asymmetry of the IHD and on the patellar displacement over the femoral diaphysis were well described in previous works. ${ }^{14,16}$ Other pelvic asymmetries may be evident and could be used for this purpose, as the medial acetabular walls symmetry; however, the authors do not know scientific studies that make an accurate correlation.

This sample of acceptable pelvic and femoral views had some slight rotation; better acceptable positioned views quality would have been achieved if a lower cut-off had been used, but in fact there is no objective criteria and it is completely dependent on the examiner judgment. However, since the technical quality of acceptable views was an inclusion criterion and the grades of rotation represent an improvement relatively to the malpositioned view, it ensured that acceptable views were always technically better than its pair. The SI and SC have some similarities in clinical information about hip congruence in normal views and are not used simultaneously in the main worldwide canine hip dysplasia scoring systems. However, it appears the inclusion of both in our study is important because the main anatomical references used for determining SC, femoral head centre and dorsal acetabular edge are located in pelvic dorsal planes with some separation, and since they are in the periphery of the image, they are subjected to greater radiographical spatial distortion when there is pelvic tilting along the long pelvic axis. ${ }^{21}$ In fact, in this study SC was the variable with bigger size effect in pelvic tilting set.

The results on NA, SI and SC are in agreement with other authors regarding the direct influence of pelvic tilting along the long axis on the relationship between femoral head and acetabulum, with a negative and positive effect on the projected hip congruence of underside and upperside respectively. ${ }^{10,11,22}$ Previous work performed in cadavers showed similar NA, SI and SC changes with pelvic tilting but was not significant. ${ }^{16}$ These divergences of significance between works may be understood by using different statistical tests, the use of a larger sample and higher mean pelvic tilting. A fact consistent with the results of similar work on cadavers is the magnitude of improvement in the variables is greater on the upperside than the worsening of the same variables on the underside. Thus, the mean NA in the underside was lower but not significant. Consequently, pelvic tilting may impair the final classifications in hip dysplasia scoring systems that use the dog's poorest articulation (i.e. $\mathrm{FCI}$ ) and benefit systems that use both joints (i.e. BVA/KC). ${ }^{1,10}$ The difference of $1.6^{\circ}$ (although not significant) in NA between underside and upperside in acceptable positioned pelvis set was not expected. Greater symmetry in NA was not achieved because there was some rotation bias of acceptable positioned pelvic views; approximately $60 \%$ maintained some level of tilting to the same side as the malpositioned view.

The estimation of the internal and external femoral rotation based on the patellar displacement within the distal femoral metaphysis is well described in a previous work. ${ }^{14}$ The internal or external femoral rotation and the absence of significant effects on NA, SI and SC were not expected, since they do not agree with the results in a previous work on cadavers. ${ }^{14}$ The 
authors' explanation for this divergence is that in clinical practice, internal femoral rotation positioning in malpositioned views is often accompanied by simultaneous adduction of the hindlimbs which promotes subluxation of hip, ${ }^{15,23}$ and the possible beneficial effects of internal rotation on hip congruence are not evident. On the other hand, internal femoral rotation in clinical practice may not arise from an excess of examiner pronation force, but from flaccidity of the periarticular soft tissues, so it may not promote joint congruence. In a cadaveric model, internal femoral rotation may have been achieved by the application of an excess of force so the femoral neck axis acts as a fulcrum promoting joint congruence. ${ }^{14}$ Although hindlimb adduction and subluxation of the hip are recognized, there are no scientific studies that associate it with variables such as NA, SI or SC.

External femoral rotation can be seen to alter the relationship between the femoral head and acetabulum in cadaveric studies ${ }^{14}$ but was not identified in this study, as often in clinical practice, external femoral rotation is accompanied by simultaneous hindlimb abduction which promotes hip congruence. ${ }^{17,23}$ On the other hand, external femoral rotation occurs in dogs with great muscle mass development, so that normal examiner force results in good hip congruence, despite the absence of femoral pronation. The application of great examiner force to obtain an acceptable view in the second radiographic examination promotes additional femoral pronation but not more hip congruence. Femoral abduction masks joint laxity and improves congruity but there are no scientific studies that associate it with variables such as NA, SI or SC.

Because the degree of femoral abduction and adduction in the malpositioned femoral rotation sets was not quantified in this study, and the absence of studies correlating its interaction with hip congruence can be mentioned as a strong limitation of this study.

\section{Conclusions}

In practice, pelvic tilting along the long axis in the VDHE view results in hip NA, SI and SC changes, non-favourable on underside and favourable on upperside for canine hip dysplasia scoring, being of greater magnitude on upperside. The $\mathrm{SC}$ is the parameter more affected by pelvic tilting.

In practice, internal or external femoral rotation did not change NA, SI and SC.

\section{Author Contribution}

J. Martins contributed to conception and design, acquisition of data, data analysis and interpretation, drafting of the article, approving submission, agreeing to be accountable for all relevant content of the work. B. Colaço contributed to conception and design, acquisition of data, data analysis and interpretation, revising the manuscript, approving the submission, agreeing to be accountable for all relevant content of the work. S. Alves-Pimenta contributed to conception and design, acquisition of data, data analysis and interpretation, revising the manuscript, approving the submission, agreeing to be accountable for all relevant content of the work. J.
Gonzalo Orden contributed to conception and design, acquisition of data, data analysis and interpretation, revising the manuscript, approving the submission, agreeing to be accountable for all relevant content of the work. A. Ferreira contributed to conception and design, acquisition of data, data analysis and interpretation, revising the manuscript, approving the submission, agreeing to be accountable for all relevant content of the work. M. Ginja contributed to conception and design, acquisition of data, data analysis and interpretation, revising the manuscript, approving the submission, agreeing to be accountable for all relevant content of the work.

\section{Animal Care}

Due to the observational and retrospective nature of the study, the ethical committee approval and the owner consent were waived.

\section{Conflict of Interest}

The authors declare no conflict of interest related to this report.

\section{Acknowledgments}

This work is supported by National Funds by FCT-Portuguese Foundation for Science and Technology, under the project UID/AGR/04033/2019.

\section{References}

1 Ginja MM, Silvestre AM, Gonzalo-Orden JM, Ferreira AJ. Diagnosis, genetic control and preventive management of canine hip dysplasia: a review. Vet J 2010;184(03):269-276

2 Ginja M, Gaspar AR, Ginja C. Emerging insights into the genetic basis of canine hip dysplasia. Vet Med (Auckl) 2015;6:193-202

3 Whittington K, Banks WC, Carlson WD, et al. Report of panel on canine hip dysplasia. J Am Vet Med Assoc 1961;139:791-806

4 Andronescu AA, Kelly L, Kearney MT, Lopez MJ. Associations between early radiographic and computed tomographic measures and canine hip joint osteoarthritis at maturity. Am J Vet Res 2015;76(01):19-27

5 Kirberger RM. Phenotypic hip and elbow dysplasia trends in Rottweilers and Labrador retrievers in South Africa (20072015): are we making progress? J S Afr Vet Assoc 2017;88(00): e1-e10

6 Worth AJ, Cave NJ. A veterinary perspective on preventing injuries and other problems that shorten the life of working dogs. Rev Sci Tech 2018;37(01):161-169

7 Corley EA. Role of the orthopedic foundation for animals in the control of canine hip dysplasia. Vet Clin North Am Small Anim Pract 1992;22(03):579-593

8 Verhoeven G, Coopman F, Duchateau L, Saunders JH, van Rijssen B, van Bree $H$. Interobserver agreement in the diagnosis of canine hip dysplasia using the standard ventrodorsal hip-extended radiographic method. J Small Anim Pract 2007;48(07):387-393

9 Ginja MM, Silvestre AM, Colaço J, et al. Hip dysplasia in Estrela mountain dogs: prevalence and genetic trends 1991-2005. Vet J 2009;182(02):275-282

10 Dennis R. Interpretation and use of BVA/KC hip scores in dogs. In Pract 2012;34:178-194

11 Genevois JP, Cachon T, Fau D, et al. Canine hip dysplasia radiographic screening. Prevalence of rotation of the pelvis along its length axis in 7,012 conventional hip extended radiographs. Vet Comp Orthop Traumatol 2007;20(04):296-298 
12 Martins J, Colaço BJ, Alves-Pimenta S, Ferreira AJ, Ginja MM. Effects of pelvis rotation on projected radiographic position of femoral head in relationship to acetabulum. Vet Med (Czech) 2017;62:377-385

13 Bausman JA, Wendelburg KL. Evaluation of the effect of pelvic tilt in the coronal plane on the Norberg angle measured in ventrodorsal radiographic views of a canine hip joint bone model. Am J Vet Res 2010;71(11):1348-1353

14 Martins J, Colaço BJ, Alves-Pimenta S, Gonzalo-Orden JM, Ferreira AJ, Ginja MM. Femoral rotation and relationship between the femoral head and the acetabulum. Vet Med (Czech) 2017;62:589-595

$15 \mathrm{Wu} \mathrm{CH}$, Lin CC, Lu Hl, Lu TW, Yeh LS. Effects of pelvic and femoral positioning on canine Norberg angle measurements and test-retest reliability: a computed tomography-based simulation study. Biomed Eng Appl Basis Commun 2014;26:14500761-145007618

16 Martins J, Colaço BJ, Ferreira AJ, Ginja MM. Analysis of pelvic rotation on the standard hip ventrodorsal extended radiographic view. Vet Comp Orthop Traumatol 2016;29(01):68-74

17 Henricson B, Norberg I, Olsson SE. On the etiology and pathogenesis of hip dysplasia: a comparative review. J Small Anim Pract 1966;7(11):673-688
18 Gaspar AR, Hayes G, Ginja C, Ginja MM, Todhunter RJ. The Norberg angle is not an accurate predictor of canine hip conformation based on the distraction index and the dorsolateral subluxation score. Prev Vet Med 2016;135:47-52

19 Flückiger MA, Friedrich GA, Binder H. A radiographic stress technique for evaluation of coxofemoral joint laxity in dogs. Vet Surg 1999;28(01):1-9

20 Cohen J. Statistical Power Analysis for the Behavioral Sciences. 2nd ed. London: Lawrence Erlbaum Associates, Inc.; 1988:19-66

21 Mixdorf M, Tortorici M. Geometric image quality. In: Tortorici M, ed. Concepts in Medical Radiographic Imaging: Circuitry, Exposure and Quality Control. Philadelphia: WB Saunders; 1992: 310-323

22 Thompson R, Roe SC, Robertson ID. Effects of pelvic positioning and simulated dorsal acetabular rim remodeling on the radiographic shape of the dorsal acetabular edge. Vet Radiol Ultrasound 2007;48(01):8-13

23 Ginja MM, Gonzalo-Orden JM, Melo-Pinto P, et al. Early hip laxity examination in predicting moderate and severe hip dysplasia in Estrela mountain dog. J Small Anim Pract 2008; 49(12):641-646 